\title{
Rota-Baxter Operators on 3-Dimensional Lie Algebras and the Classical $R$-Matrices
}

\author{
Linli Wu, Mengping Wang, and Yongsheng Cheng \\ School of Mathematics and Statistics, Henan University, Kaifeng 475004, China \\ Correspondence should be addressed to Yongsheng Cheng; yscheng@henu.edu.cn
}

Received 6 September 2017; Accepted 27 November 2017; Published 18 December 2017

Academic Editor: Boris G. Konopelchenko

Copyright (C) 2017 Linli Wu et al. This is an open access article distributed under the Creative Commons Attribution License, which permits unrestricted use, distribution, and reproduction in any medium, provided the original work is properly cited.

Our aim is to classify the Rota-Baxter operators of weight 0 on the 3-dimensional Lie algebra whose derived algebra's dimension is 2. We explicitly determine all Rota-Baxter operators (of weight zero) on the 3-dimensional Lie algebras $g$. Furthermore, we give the corresponding solutions of the classical Yang-Baxter equation in the 6-dimensional Lie algebras $g \ltimes_{\mathrm{ad}^{*}} g^{*}$ and the induced left-symmetry algebra structures on $g$.

\section{Introduction}

In physics, the Yang-Baxter equation is a consistency equation which was first introduced in the field of statistical mechanics. It depends on the idea that, in some scattering situations, particles may preserve their momentum while changing their quantum internal states. Rota-Baxter algebra started with the probability study and has since found applications in many areas of mathematics and physics, such as quasi-symmetric functions, number theory, dendriform algebras, and Yang-Baxter equations.

A Rota-Baxter operator (of weight zero) on an associative algebra $A$ is defined to be a linear map $P: g \rightarrow g$ satisfying

$$
P(x) P(y)=P(P(x) y+x P(y)), \quad \forall x, y \in A .
$$

Rota-Baxter operators (on associative algebras) were introduced by Baxter to solve an analytic formula in probability [1-4]. It has been related to other areas in mathematics and mathematical physics [5-9]. A Rota-Baxter operator (of weight zero) on a Lie algebra $(g,[\cdot, \cdot])$ is a linear operator $P: g \rightarrow g$ such that

$$
\begin{array}{r}
{[P(x), P(y)]=P([P(x), y]+[x, P(y)]),} \\
\forall x, y \in g .
\end{array}
$$

In fact, a Rota-Baxter operator is also called the operator form of the classical Yang-Baxter equation [10-13]. Let $g$ be a
Lie algebra and $r=\sum_{i} a_{i} \otimes b_{i} \in g \otimes g$. $r$ is called a classical $R$ matrix if it is a solution of the classical Yang-Baxter equation (CYBE) in $g$ : that is,

$$
\left[r_{12}, r_{13}\right]+\left[r_{12}, r_{23}\right]+\left[r_{13}, r_{23}\right]=0
$$

in $U(g)$, where $U(g)$ is the universal enveloping algebra of $g$ and

$$
\begin{aligned}
& r_{12}=\sum_{i} a_{i} \otimes b_{i} \otimes 1, \\
& r_{13}=\sum_{i} a_{i} \otimes 1 \otimes b_{i}, \\
& r_{23}=\sum_{i} 1 \otimes a_{i} \otimes b_{i} .
\end{aligned}
$$

Set $r^{21}=\sum_{i} b_{i} \otimes a_{i}$. It is easy to obtain that $r$ is skew-symmetric if and only if $r=-r^{21}$. Semenov-Tian-Shansky proved in [14] that $r$ is skew-symmetric and there is a nondegenerate symmetric invariant bilinear form on Lie algebra $g$; relation (2) is equivalent to relation (3) when the weight is zero. Furthermore, Rota-Baxter operators of weights 0 and 1 on a Lie algebra $g$ give rise to solutions of CYBE on the double Lie algebra $g \ltimes_{\mathrm{ad}^{*}} g^{*}$ over the direct sum $g \oplus g^{*}$ of the Lie algebra $g$ and its dual space $g^{*}[12,15,16]$. Moreover, we can get some solutions of CYBE in $g \ltimes_{\mathrm{ad}^{*}} g^{*}$ Lie algebras through Rota-Baxter operators of any weight on $g$. 
In [12], the authors gave all Rota-Baxter operators (of weight zero) on 3-dimensional simple Lie algebra sl $(2, \mathbb{C})$. The aim of this paper is to determine the Rota-Baxter operators (of weight zero) on the 3-dimensional Lie algebra which is not simple, and the dimension of its derived algebra is 2 . We will determine the Rota-Baxter operators on the Lie algebra $g$ and give a family of solutions of CYBE in $g \ltimes_{\mathrm{ad}^{*}} g^{*}$. This paper is organized as follows. In Section 2, we give the classification theorem of Rota-Baxter operators (of weight zero) on $g$. In Section 3, we give the corresponding solutions of CYBE in $g \ltimes_{\mathrm{ad}^{*}} g^{*}$. In Section 4, we give the corresponding leftsymmetry structure on $g$.

\section{The Rota-Baxter Operators on $g$ (of Weight Zero)}

2.1. Notations and the Classification Theorem. Let $g$ be a 3dimensional linear Lie algebra whose standard (Cartan-Weyl) basis consists of $e_{1}, e_{2}, e_{3}$ over the field of complex numbers $\mathbb{C}$ with the following Lie brackets:

$$
\begin{aligned}
& {\left[e_{1}, e_{2}\right]=e_{1},} \\
& {\left[e_{1}, e_{3}\right]=0,} \\
& {\left[e_{2}, e_{3}\right]=e_{1}+e_{3} .}
\end{aligned}
$$

Thus, a linear operator $P: g \rightarrow g$ is determined by

$$
\left(\begin{array}{l}
P\left(e_{1}\right) \\
P\left(e_{2}\right) \\
P\left(e_{3}\right)
\end{array}\right)=\left(\begin{array}{lll}
b_{11} & b_{12} & b_{13} \\
b_{21} & b_{22} & b_{23} \\
b_{31} & b_{32} & b_{33}
\end{array}\right)\left(\begin{array}{l}
e_{1} \\
e_{2} \\
e_{3}
\end{array}\right)
$$

where $b_{i j} \in \mathbb{C}, 1 \leq i, j \leq 3$. $P$ is a Rota-Baxter operator on $g$ if the above matrix $\left(b_{i j}\right)_{3 \times 3}$ satisfies (2). Here is our main theorem.

Theorem 1. All Rota-Baxter operators of weight zero on g are listed in their matrices form with respect to the Cartan-Weyl basis below, where $a, b$, and $c$ are nonzero complex numbers.

$$
\begin{aligned}
& P_{1}=\left(\begin{array}{lll}
0 & 0 & 0 \\
0 & 0 & 0 \\
a & 1 & 0
\end{array}\right), \\
& P_{2}=\left(\begin{array}{ccc}
0 & 0 & 0 \\
0 & a & -a^{2} \\
0 & 1 & -a
\end{array}\right), \\
& P_{3}=\left(\begin{array}{lll}
0 & 0 & 0 \\
0 & 0 & 0 \\
0 & 1 & 0
\end{array}\right),
\end{aligned}
$$

$$
\begin{aligned}
& P_{4}=\left(\begin{array}{ccc}
0 & 0 & 0 \\
a b & a & -a^{2} \\
b & 1 & -a
\end{array}\right), \\
& P_{5}=\left(\begin{array}{lll}
0 & 0 & 0 \\
a & 0 & 1 \\
0 & 0 & 0
\end{array}\right), \\
& P_{6}=\left(\begin{array}{lll}
0 & 0 & 0 \\
1 & 0 & 0 \\
0 & 0 & 0
\end{array}\right), \\
& P_{7}=\left(\begin{array}{lll}
0 & 0 & 0 \\
0 & 0 & 1 \\
0 & 0 & 0
\end{array}\right), \\
& P_{8}=\left(\begin{array}{lll}
0 & 0 & 0 \\
0 & 0 & 0 \\
0 & 0 & 0
\end{array}\right), \\
& P_{9}=\left(\begin{array}{lll}
0 & 0 & 0 \\
a & 0 & b \\
1 & 0 & 0
\end{array}\right),
\end{aligned}
$$$$
P_{10}=\left(\begin{array}{lll}
0 & 0 & 0 \\
a & 0 & 0 \\
1 & 0 & 0
\end{array}\right)
$$$$
P_{11}=\left(\begin{array}{lll}
0 & 0 & 0 \\
0 & 0 & a \\
1 & 0 & 0
\end{array}\right) \text {, }
$$$$
P_{12}=\left(\begin{array}{ccc}
0 & 0 & 0 \\
0 & 0 & 0 \\
1 & 0 & 0
\end{array}\right) \text {, }
$$$$
P_{13}=\left(\begin{array}{ccc}
0 & 0 & 0 \\
a & 1 & b \\
0 & 0 & 0
\end{array}\right) \text {, }
$$$$
P_{14}=\left(\begin{array}{ccc}
0 & 0 & 0 \\
a & 1 & 0 \\
0 & 0 & 0
\end{array}\right) \text {, }
$$$$
P_{15}=\left(\begin{array}{lll}
0 & 0 & 0 \\
0 & 1 & a \\
0 & 0 & 0
\end{array}\right) \text {, }
$$$$
P_{16}=\left(\begin{array}{ccc}
0 & 0 & 0 \\
0 & 1 & 0 \\
0 & 0 & 0
\end{array}\right) \text {, }
$$ 


$$
\begin{aligned}
& P_{17}=\left(\begin{array}{ccc}
0 & 0 & 1 \\
a & 0 & b \\
0 & 0 & -1
\end{array}\right) \\
& P_{18}=\left(\begin{array}{ccc}
a & 0 & 1 \\
0 & 0 & b \\
a^{2}-a & 0 & a-1
\end{array}\right) \quad\left(a \neq \frac{1}{2}\right), \\
& P_{19}=\left(\begin{array}{ccc}
a & 0 & 1 \\
b & 0 & 0 \\
a^{2}-a & 0 & a-1
\end{array}\right) \quad\left(a \neq \frac{1}{2}\right) \text {, } \\
& P_{20}=\left(\begin{array}{ccc}
0 & 0 & 1 \\
0 & 0 & a \\
0 & 0 & -1
\end{array}\right) \text {, } \\
& P_{21}=\left(\begin{array}{ccc}
0 & 0 & 1 \\
a & 0 & 0 \\
0 & 0 & -1
\end{array}\right) \text {, } \\
& P_{22}=\left(\begin{array}{ccc}
a & 0 & 1 \\
0 & 0 & 0 \\
a^{2}-a & 0 & a-1
\end{array}\right) \quad\left(a \neq \frac{1}{2}\right), \\
& P_{23}=\left(\begin{array}{ccc}
0 & 0 & 1 \\
0 & 0 & 0 \\
0 & 0 & -1
\end{array}\right) \text {, } \\
& P_{24}=\left(\begin{array}{ccc}
a & 0 & 1 \\
b & 0 & c \\
a^{2}-a & 0 & a-1
\end{array}\right) \quad\left(a \neq \frac{1}{2}\right), \\
& P_{25}=\left(\begin{array}{ccc}
\frac{1}{2} & 0 & 1 \\
0 & 0 & a \\
-\frac{1}{4} & 0 & -\frac{1}{2}
\end{array}\right) \text {, } \\
& P_{26}=\left(\begin{array}{ccc}
\frac{1}{2} & 0 & 1 \\
a & 0 & 0 \\
-\frac{1}{4} & 0 & -\frac{1}{2}
\end{array}\right) \text {, } \\
& P_{27}=\left(\begin{array}{ccc}
\frac{1}{2} & 0 & 1 \\
0 & 0 & 0 \\
-\frac{1}{4} & 0 & -\frac{1}{2}
\end{array}\right) \text {, } \\
& P_{28}=\left(\begin{array}{ccc}
\frac{1}{2} & 0 & 1 \\
a & 0 & b \\
-\frac{1}{4} & 0 & -\frac{1}{2}
\end{array}\right) \text {, }
\end{aligned}
$$

$$
\begin{aligned}
P_{29} & =\left(\begin{array}{lll}
0 & 1 & 2 a \\
0 & a & 2 a^{2} \\
0 & -\frac{1}{2} & -a
\end{array}\right), \\
P_{30} & =\left(\begin{array}{ccc}
a & 1 & 2 a \\
0 & 0 & 0 \\
-\frac{a}{2} & -\frac{1}{2} & -a
\end{array}\right), \\
P_{31} & =\left(\begin{array}{ccc}
0 & 1 & 0 \\
0 & 0 & 0 \\
0 & -\frac{1}{2} & 0
\end{array}\right), \\
P_{32} & =\left(\begin{array}{ccc}
a & 1 & 2(a+b) \\
a b & b & -2 b^{2}-2 a b \\
-\frac{a}{2} & -\frac{1}{2} & -(a+b)
\end{array}\right) .
\end{aligned}
$$

2.2. Reduction to Quadratic Equations. In order to show that $P$ is a Rota-Baxter operator, we only check the following:

$$
\begin{aligned}
& {\left[P\left(e_{1}\right), P\left(e_{2}\right)\right]=P\left(\left[P\left(e_{1}\right), e_{2}\right]+e_{1}, P\left(e_{2}\right)\right],} \\
& {\left[P\left(e_{2}\right), P\left(e_{3}\right)\right]=P\left(\left[P\left(e_{2}\right), e_{3}\right]+e_{2}, P\left(e_{3}\right)\right],} \\
& {\left[P\left(e_{1}\right), P\left(e_{3}\right)\right]=P\left(\left[P\left(e_{1}\right), e_{3}\right]+e_{1}, P\left(e_{3}\right)\right] .}
\end{aligned}
$$

It follows from (5) and (6) that

$$
\begin{aligned}
{\left[P\left(e_{2}\right), P\left(e_{3}\right)\right]=} & \left(b_{21} b_{32}-b_{22} b_{31}+b_{22} b_{33}-b_{23} b_{32}\right) e_{1} \\
& +\left(b_{22} b_{33}-b_{23} b_{32}\right) e_{3},
\end{aligned}
$$

while

$$
\begin{aligned}
P( & {\left.\left[P\left(e_{2}\right), e_{3}\right]+\left[e_{2}, P\left(e_{3}\right)\right]\right) } \\
= & P\left(\left(b_{22}-b_{31}+b_{33}\right) e_{1}\right)-P\left(\left(b_{22}+b_{33}\right) e_{3}\right) \\
= & \left(b_{22} b_{11}-b_{31} b_{11}+b_{33} b_{11}+b_{22} b_{31}+b_{33} b_{31}\right) e_{1} \\
& +\left(b_{22} b_{12}-b_{31} b_{12}+b_{33} b_{12}+b_{22} b_{32}+b_{33} b_{32}\right) e_{2} \\
& +\left(b_{22} b_{13}-b_{31} b_{13}+b_{33} b_{13}+b_{22} b_{33}+b_{33} b_{33}\right) e_{3} .
\end{aligned}
$$

Comparing the coefficients in (9) and (10), we have

$$
\begin{aligned}
& b_{21} b_{32}-2 b_{22} b_{31}+b_{22} b_{33}-b_{23} b_{32}-b_{22} b_{11}+b_{31} b_{11} \\
& \quad-b_{33} b_{11}-b_{33} b_{31}=0 \\
& b_{22} b_{12}-b_{31} b_{12}+b_{33} b_{12}+b_{22} b_{32}+b_{33} b_{32}=0 \\
& b_{23} b_{32}+b_{22} b_{13}-b_{31} b_{13}+b_{33} b_{13}+b_{33} b_{33}=0 .
\end{aligned}
$$

Similarly, from

$$
\begin{aligned}
& {\left[P\left(e_{1}\right), P\left(e_{2}\right)\right]=P\left(\left[P\left(e_{1}\right), e_{2}\right]+e_{1}, P\left(e_{2}\right)\right],} \\
& {\left[P\left(e_{1}\right), P\left(e_{3}\right)\right]=P\left(\left[P\left(e_{1}\right), e_{3}\right]+e_{1}, P\left(e_{3}\right)\right],}
\end{aligned}
$$


we obtain the following six equations:

$$
\begin{aligned}
b_{11} b_{11}+b_{12} b_{21}-b_{13} b_{11}-b_{13} b_{31}-b_{12} b_{23}+b_{13} b_{22} & =0 \\
b_{11} b_{12}+b_{22} b_{12}-b_{13} b_{12}-b_{13} b_{32} & =0 \\
b_{11} b_{13}+2 b_{22} b_{13}-b_{13} b_{13}-b_{13} b_{33}-b_{12} b_{23} & =0 \\
b_{12} b_{33}-2 b_{12} b_{31}-b_{13} b_{32}-b_{12} b_{11} & =0 \\
b_{12} b_{12}+2 b_{32} b_{12} & =0 \\
b_{12} b_{13}+2 b_{32} b_{13} & =0 .
\end{aligned}
$$

2.3. Solving the Quadratic Equations. Equation (19) implies $b_{12}\left(b_{12}+2 b_{32}\right)=0$. To solve the quadratic equations (11), (12), (13), (15), (16), (17), (18), (19), and (20), we distinguish the following cases depending on whether $b_{12}=0$ or not.

Case 1. $b_{12}=0, b_{12}+2 b_{32} \neq 0$. That is, $b_{12}=0, b_{32} \neq 0$, taking $b_{32}=1$. Equation (16) implies $b_{13}=0$. Equation (15) implies $b_{11}=0$. Equation (11) implies $b_{21}=b_{22} b_{31}+b_{22}^{2}+b_{23}$. Equation (12) implies $b_{33}=-b_{22}$. Equation (13) implies $b_{23}=-b_{33}^{2}=$ $-b_{22}^{2}$. We obtain

$$
P=\left(\begin{array}{ccc}
0 & 0 & 0 \\
b_{22} b_{31} & b_{22} & -b_{22}^{2} \\
b_{31} & 1 & -b_{22}
\end{array}\right)
$$

Taking $b_{22}=0, b_{31}=a$, we obtain $P_{1}$. Taking $b_{22}=a, b_{31}=0$, we obtain $P_{2}$. Taking $b_{22}=0, b_{31}=0$, we obtain $P_{3}$. Taking $b_{22}=a, b_{31}=b$, we obtain $P_{4}$.

Case 2. Assume $b_{12}=0, b_{12}+2 b_{32}=0$. That is, $b_{12}=0, b_{32}=0$. We distinguish the two cases depending on whether $b_{13}=0$ or not.

Subcase 2.1. If $b_{13}=0$, then (13) implies $b_{33}=0$. Equation (15) implies $b_{11}=0$. Equation (11) implies $b_{22} b_{31}=0$.

Subcase 2.1.1. If $b_{22}=0, b_{31}=0$, we obtain

$$
P=\left(\begin{array}{ccc}
0 & 0 & 0 \\
b_{21} & 0 & b_{23} \\
0 & 0 & 0
\end{array}\right) \text {. }
$$

Taking $b_{21}=a, b_{23}=1$, we obtain $P_{5}$. Taking $b_{21}=1, b_{23}=0$, we obtain $P_{6}$. Taking $b_{21}=0, b_{23}=1$, we obtain $P_{7}$. Taking $b_{21}=0, b_{23}=0$, we obtain $P_{8}$.

Subcase 2.1.2. If $b_{22}=0, b_{31} \neq 0$, taking $b_{31}=1$, we obtain

$$
P=\left(\begin{array}{ccc}
0 & 0 & 0 \\
b_{21} & 0 & b_{23} \\
1 & 0 & 0
\end{array}\right) \text {. }
$$

Taking $b_{21}=a, b_{23}=b$, we obtain $P_{9}$. Taking $b_{21}=a, b_{23}=0$, we obtain $P_{10}$. Taking $b_{21}=0, b_{23}=a$, we obtain $P_{11}$. Taking $b_{21}=0, b_{23}=0$, we obtain $P_{12}$.
Subcase 2.1.3. If $b_{22} \neq 0, b_{31}=0$, taking $b_{22}=1$, we obtain

$$
P=\left(\begin{array}{ccc}
0 & 0 & 0 \\
b_{21} & 1 & b_{23} \\
0 & 0 & 0
\end{array}\right)
$$

Taking $b_{21}=a, b_{23}=b$, we obtain $P_{13}$. Taking $b_{21}=a, b_{23}=a$, we obtain $P_{14}$. Taking $b_{21}=0, b_{23}=a$, we obtain $P_{15}$. Taking $b_{21}=0, b_{23}=0$, we obtain $P_{16}$.

Subcase 2.2. If $b_{13} \neq 0$, taking $b_{13}=1$, (17) implies $b_{22}=$ $\left(b_{33}-b_{11}+1\right) / 2$. Then (15) implies $b_{31}=\left(2 b_{11}^{2}-3 b_{11}+b_{33}+\right.$ $1) / 2$. Equation (13) implies $b_{11}+b_{33}=b_{11}^{2}-b_{33}^{2}$. That is, $\left(b_{11}+\right.$ $\left.b_{33}\right)\left(b_{11}-b_{33}-1\right)=0$.

Subcase 2.2.1. If $b_{11}+b_{33} \neq 0, b_{11}-b_{33}-1=0,\left(b_{11} \neq 1 / 2\right)$, and then $b_{22}=0, b_{31}=b_{11}^{2}-b_{11}$, we obtain

$$
P=\left(\begin{array}{ccc}
b_{11} & 0 & 1 \\
b_{21} & 0 & b_{23} \\
b_{11}^{2}-b_{11} & 0 & b_{11}-1
\end{array}\right), \quad\left(b_{11} \neq \frac{1}{2}\right)
$$

Taking $b_{11}=0, b_{21}=a, b_{23}=b$, we obtain $P_{17}$. Taking $b_{11}=a$, $b_{21}=0, b_{23}=b(a \neq 1 / 2)$, we obtain $P_{18}$. Taking $b_{11}=a$, $b_{21}=b, b_{23}=0(a \neq 1 / 2)$, we obtain $P_{19}$. Taking $b_{11}=0$, $b_{21}=0, b_{23}=a$, we obtain $P_{20}$. Taking $b_{11}=0, b_{21}=a, b_{23}=0$, we obtain $P_{21}$. Taking $b_{11}=a, b_{21}=0, b_{23}=0(a \neq 1 / 2)$, we obtain $P_{22}$. Taking $b_{11}=0, b_{21}=0, b_{23}=0$, we obtain $P_{23}$. Taking $b_{11}=a, b_{21}=b, b_{23}=c(a \neq 1 / 2)$, we obtain $P_{24}$.

Subcase 2.2.2. If $b_{11}+b_{33}=0, b_{11}-b_{33}-1 \neq 0\left(b_{11} \neq 1 / 2\right)$, and then $b_{22}=\left(-2 b_{11}+1\right) / 2, b_{31}=\left(2 b_{11}^{2}-4 b_{11}+1\right) / 2,(3.4)$ implies $8 b_{11}^{3}-12 b_{11}^{2}+6 b_{11}-1=0$. Then we have $8\left(b_{11}-1 / 2\right)^{3}=0$, $b_{11}=1 / 2$, giving a contradiction.

Subcase 2.2.3. If $b_{11}+b_{33}=0, b_{11}-b_{33}-1=0$, that is, $b_{11}=1 / 2$, $b_{33}=-1 / 2$, and then $b_{22}=0, b_{31}=-1 / 4$, we obtain

$$
P=\left(\begin{array}{ccc}
\frac{1}{2} & 0 & 1 \\
b_{21} & 0 & b_{23} \\
-\frac{1}{4} & 0 & -\frac{1}{2}
\end{array}\right)
$$

Taking $b_{21}=0, b_{23}=a$, we obtain $P_{25}$. Taking $b_{21}=a, b_{23}=0$, we obtain $P_{26}$. Taking $b_{21}=0, b_{23}=0$, we obtain $P_{27}$. Taking $b_{21}=a, b_{23}=b$, we obtain $P_{28}$.

Case 3. Assume $b_{12} \neq 0,\left(b_{12}+2 b_{32}\right)=0$. Taking $b_{12}=1$, then $b_{32}=-1 / 2$. Equation (16) implies $b_{13}=2 b_{11}+2 b_{22}$. Equations (12) and (18) imply $b_{31}=\left(b_{22}+b_{33}\right) / 2$. Equation (17) implies $b_{23}=-2 b_{11}^{2}-2 b_{11} b_{22}-2 b_{11} b_{33}-2 b_{22} b_{33}$. Equation (15) implies $b_{21}=-b_{11} b_{22}-b_{11} b_{33}-b_{22} b_{33}-b_{11}^{2}-b_{22}^{2}$. Equations (11), (15), and (17) imply $b_{11}^{2}+b_{22}^{2}+b_{33}^{2}+2 b_{11} b_{22}+2 b_{11} b_{33}+2 b_{22} b_{33}=0$. 
Then we have $\left(b_{11}+b_{22}+b_{33}\right)^{2}=0$. So $b_{33}=-\left(b_{11}+b_{22}\right)$, $b_{21}=b_{11} b_{22}, b_{23}=-2 b_{22}^{2}-2 b_{11} b_{22}, b_{31}=-b_{11} / 2$. We obtain

$$
P=\left(\begin{array}{ccc}
b_{11} & 1 & 2 b_{11}+2 b_{22} \\
b_{11} b_{22} & b_{22} & -2 b_{22}^{2}-2 b_{11} b_{22} \\
-\frac{b_{11}}{2} & -\frac{1}{2} & -\left(b_{11}+b_{22}\right)
\end{array}\right)
$$

Taking $b_{11}=0, b_{22}=a$, we obtain $P_{29}$. Taking $b_{11}=a, b_{22}=0$, we obtain $P_{30}$. Taking $b_{11}=0, b_{22}=0$, we obtain $P_{31}$. Taking $b_{11}=a, b_{22}=b$, we obtain $P_{32}$.

\section{Solutions of the CYBE in $g \ltimes_{a d^{*}} g^{*}$}

In this section, we will give some solutions of CYBE in $g \ltimes_{\mathrm{ad}^{*}} g^{*}$. Let $(g,[]$,$) be a Lie algebra and \beta: g \rightarrow g l(V)$ a representation of $g$. On the vector space $g \oplus V$, there is natural Lie algebra structure (denoted by $g \ltimes_{\beta} V$ ) given by

$$
\begin{array}{r}
{\left[x_{1}+v_{1}, x_{2}+v_{2}\right]=\left[x_{1}, x_{2}\right]+\beta\left(x_{1}\right) v_{2}-\beta\left(x_{2}\right) v_{1}} \\
x_{1}, x_{2} \in g, v_{1}, v_{2} \in V .
\end{array}
$$

Let $\beta^{*}: g \rightarrow g l\left(V^{*}\right)$ be the dual representation of $\beta$. A linear map $P: V \rightarrow g$ can be identified as an element $\widetilde{P}$ in $g \otimes$ $V^{*} \subset\left(g \ltimes_{\beta}^{*} V^{*}\right) \otimes\left(g \ltimes_{\beta}^{*} V^{*}\right)$ as follows. Let $\left\{v_{1}, v_{2}, \ldots, v_{m}\right\}$ be a basis of $V$ and $\left\{v_{1}^{*}, v_{2}^{*}, \ldots, v_{m}^{*}\right\}$ the dual basis in $V^{*}$ : that is, $v_{i}^{*}\left(v_{j}\right)=\delta_{i j}$. Let $\left\{e_{1}, e_{2}, \ldots, e_{n}\right\}$ be a basis of $g$. Set $P\left(v_{i}\right)=$ $\sum_{i=1}^{n} a_{i j} e_{j}, \quad 1 \leq i \leq n$. Since, as a vector space, $\operatorname{Hom}(V, g) \cong$ $g \otimes V^{*}$, then

$$
\begin{aligned}
\widetilde{P} & =\sum_{i=1}^{n} P\left(v_{i}\right) \otimes v_{i}^{*}=\sum_{i=1}^{m} \sum_{j=1}^{n} a_{i j} e_{j} \otimes v_{i}^{*} \\
& \subseteq\left(g \ltimes_{\beta}^{*} V^{*}\right) \otimes\left(g \ltimes_{\beta}^{*} V^{*}\right) .
\end{aligned}
$$

Lemma 2 (see [15]). Let $g$ be a Lie algebra; let $(V, \beta)$ be a $g$ module. A linear map $P: g \rightarrow g$ is a Rota-Baxter operator if and only if $r=P-P^{21}$ is a skew-symmetric solution of CYBE in $g \ltimes_{a d^{*}} g^{*}$.

Now consider the adjoint representation of $g,(g, a d)$ which is a $g$-module. Let $e_{1}, e_{2}, e_{3}$ be the Cartan-Weyl basis. Using Lemma 2 and relation (29), we can obtain a family of solutions of CYBE in $g \ltimes_{\mathrm{ad}^{*}} g^{*}$ through the Rota-Baxter operators on $g$ given in Theorem 1 .

Theorem 3. The following tensors are solutions of the classical Yang-Baxter equation in $g \ltimes_{a d^{*}} g^{*}$, where $a, b$, and $c$ are nonzero complex numbers

$$
\begin{aligned}
r_{1} & =\left(a e_{1}+e_{2}\right) \otimes e_{3}^{*}-e_{3}^{*} \otimes\left(a e_{1}+e_{2}\right), \\
r_{2} & =\left(a e_{2}-a^{2} e_{3}\right) \otimes e_{2}^{*}+\left(e_{2}-a e_{3}\right) \otimes e_{3}^{*}-e_{2}^{*} \\
& \otimes\left(a e_{2}-a^{2} e_{3}\right)-e_{3}^{*} \otimes\left(e_{2}-a e_{3}\right),
\end{aligned}
$$

$$
\begin{aligned}
& r_{3}=e_{2} \otimes e_{3}^{*}-e_{3}^{*} \otimes e_{2}, \\
& r_{4}=\left(a b e_{1}+a e_{2}-a^{2} e_{3}\right) \otimes e_{2}^{*}+\left(b e_{1}+e_{2}-a e_{3}\right) \otimes e_{3}^{*} \\
& -e_{2}^{*} \otimes\left(a b e_{1}+a e_{2}-a^{2} e_{3}\right)-e_{3}^{*} \\
& \otimes\left(b e_{1}+e_{2}-a e_{3}\right) \text {, } \\
& r_{5}=\left(a e_{1}+e_{3}\right) \otimes e_{2}^{*}-e_{2}^{*} \otimes\left(a e_{1}+e_{3}\right), \\
& r_{6}=e_{1} \otimes e_{2}^{*}-e_{2}^{*} \otimes e_{1}, \\
& r_{7}=e_{3} \otimes e_{2}^{*}-e_{2}^{*} \otimes e_{3}^{\prime}, \\
& r_{8}=0 \text {, } \\
& r_{9}=\left(a e_{1}+b e_{3}\right) \otimes e_{2}^{*}+e_{1} \otimes e_{3}^{*}-e_{2}^{*} \otimes\left(a e_{1}+b e_{3}\right) \\
& -e_{3}^{*} \otimes e_{1} \\
& r_{10}=a e_{1} \otimes e_{2}^{*}+e_{1} \otimes e_{3}^{*}-e_{2}^{*} \otimes a e_{1}-e_{3}^{*} \otimes e_{1}, \\
& r_{11}=a e_{3} \otimes e_{2}^{*}+e_{1} \otimes e_{3}^{*}-e_{2}^{*} \otimes a e_{3}-e_{3}^{*} \otimes e_{1}, \\
& r_{12}=e_{1} \otimes e_{3}^{*}-e_{3}^{*} \otimes e_{1}, \\
& r_{13}=\left(a e_{1}+e_{2}+b e_{3}\right) \otimes e_{2}^{*}-e_{2}^{*} \otimes\left(a e_{1}+e_{2}+b e_{3}\right), \\
& r_{14}=\left(a e_{1}+e_{2}\right) \otimes e_{2}^{*}-e_{2}^{*} \otimes\left(a e_{1}+e_{2}\right), \\
& r_{15}=\left(e_{2}+a e_{3}\right) \otimes e_{2}^{*}-e_{2}^{*} \otimes\left(e_{2}+a e_{3}\right), \\
& r_{16}=e_{2} \otimes e_{2}^{*}-e_{2}^{*} \otimes e_{2}, \\
& r_{17}=e_{3} \otimes e_{1}^{*}+\left(a e_{1}+b e_{3}\right) \otimes e_{2}^{*}-e_{3} \otimes e_{3}^{*} \\
& -e_{1}^{*} \otimes e_{3}-e_{2}^{*} \otimes\left(a e_{1}+b e_{3}\right)+e_{3}^{*} \otimes e_{3}, \\
& r_{18}=\left(a e_{1}+e_{3}\right) \otimes e_{1}^{*}+b e_{3} \otimes e_{2}^{*} \\
& +\left(\left(a^{2}-a\right) e_{1}+(a-1) e_{3}\right) \otimes e_{3}^{*}-e_{1}^{*} \otimes\left(a e_{1}+e_{3}\right) \\
& -e_{2}^{*} \otimes b e_{3}-e_{3}^{*} \otimes\left(\left(a^{2}-a\right) e_{1}+(a-1) e_{3}\right) \text {, } \\
& \left(a \neq \frac{1}{2}\right), \\
& r_{19}=\left(a e_{1}+e_{3}\right) \otimes e_{1}^{*}+b e_{1} \otimes e_{2}^{*} \\
& +\left(\left(a^{2}-a\right) e_{1}+(a-1) e_{3}\right) \otimes e_{3}^{*}-e_{1}^{*} \otimes\left(a e_{1}+e_{3}\right) \\
& -e_{2}^{*} \otimes b e_{1}-e_{3}^{*} \otimes\left(\left(a^{2}-a\right) e_{1}+(a-1) e_{3}\right), \\
& \left(a \neq \frac{1}{2}\right), \\
& r_{20}=e_{3} \otimes e_{1}^{*}+a e_{3} \otimes e_{2}^{*}-e_{3} \otimes e_{3}^{*}-e_{1}^{*} \otimes e_{3}-e_{2}^{*} \\
& \otimes a e_{3}+e_{3}^{*} \otimes e_{3}, \\
& r_{21}=e_{3} \otimes e_{1}^{*}+a e_{1} \otimes e_{2}^{*}-e_{3} \otimes e_{3}^{*}-e_{1}^{*} \otimes e_{3}-e_{2}^{*} \\
& \otimes a e_{1}+e_{3}^{*} \otimes e_{3},
\end{aligned}
$$




$$
\begin{aligned}
& r_{22}=\left(a e_{1}+e_{3}\right) \otimes e_{1}^{*}+\left(\left(a^{2}-a\right) e_{1}+(a-1) e_{3}\right) \otimes e_{3}^{*} \\
& -e_{1}^{*} \otimes\left(a e_{1}+e_{3}\right)-e_{3}^{*} \otimes\left(\left(a^{2}-a\right) e_{1}+(a-1) e_{3}\right) \\
& \left(a \neq \frac{1}{2}\right) \\
& r_{23}=e_{3} \otimes e_{1}^{*}-e_{3} \otimes e_{3}^{*}-e_{1}^{*} \otimes e_{3}+e_{3}^{*} \otimes e_{3}, \\
& r_{24}=\left(a e_{1}+e_{3}\right) \otimes e_{1}^{*}+\left(b e_{1}+c e_{3}\right) \otimes e_{2}^{*} \\
& +\left(\left(a^{2}-a\right) e_{1}+(a-1) e_{3}\right) \otimes e_{3}^{*}-e_{1}^{*} \otimes\left(a e_{1}+e_{3}\right) \\
& -e_{2}^{*} \otimes\left(b e_{1}+c e_{3}\right)-e_{3}^{*} \\
& \otimes\left(\left(a^{2}-a\right) e_{1}+(a-1) e_{3}\right), \quad\left(a \neq \frac{1}{2}\right), \\
& r_{25}=\left(\frac{1}{2} e_{1}+e_{3}\right) \otimes e_{1}^{*}+a e_{3} \otimes e_{2}^{*}-\left(\frac{1}{4} e_{1}+\frac{1}{2} e_{3}\right) \\
& \otimes e_{3}^{*}-e_{1}^{*} \otimes\left(\frac{1}{2} e_{1}+e_{3}\right)-e_{2}^{*} \otimes a e_{3}+e_{3}^{*} \\
& \otimes\left(\frac{1}{4} e_{1}+\frac{1}{2} e_{3}\right) \\
& r_{26}=\left(\frac{1}{2} e_{1}+e_{3}\right) \otimes e_{1}^{*}+a e_{1} \otimes e_{2}^{*}-\left(\frac{1}{4} e_{1}+\frac{1}{2} e_{3}\right) \\
& \otimes e_{3}^{*}-e_{1}^{*} \otimes\left(\frac{1}{2} e_{1}+e_{3}\right)-e_{2}^{*} \otimes a e_{1}+e_{3}^{*} \\
& \otimes\left(\frac{1}{4} e_{1}+\frac{1}{2} e_{3}\right) \\
& r_{27}=\left(\frac{1}{2} e_{1}+e_{3}\right) \otimes e_{1}^{*}-\left(\frac{1}{4} e_{1}+\frac{1}{2} e_{3}\right) \otimes e_{3}^{*}-e_{1}^{*} \\
& \otimes\left(\frac{1}{2} e_{1}+e_{3}\right)+e_{3}^{*} \otimes\left(\frac{1}{4} e_{1}+\frac{1}{2} e_{3}\right), \\
& r_{28}=\left(\frac{1}{2} e_{1}+e_{3}\right) \otimes e_{1}^{*}+\left(a e_{1}+b e_{3}\right) \otimes e_{2}^{*} \\
& -\left(\frac{1}{4} e_{1}+\frac{1}{2} e_{3}\right) \otimes e_{3}^{*}-e_{1}^{*} \otimes\left(\frac{1}{2} e_{1}+e_{3}\right)-e_{2}^{*} \\
& \otimes\left(a e_{1}+b e_{3}\right)+e_{3}^{*} \otimes\left(\frac{1}{4} e_{1}+\frac{1}{2} e_{3}\right), \\
& r_{29}=\left(e_{2}+2 a e_{3}\right) \otimes e_{1}^{*}+\left(a e_{2}+2 a^{2} e_{3}\right) \otimes e_{2}^{*} \\
& -\left(\frac{1}{2} e_{2}+a e_{3}\right) \otimes e_{3}^{*}-e_{1}^{*} \otimes\left(e_{2}+2 a e_{3}\right)-e_{2}^{*} \\
& \otimes\left(a e_{2}+2 a^{2} e_{3}\right)+e_{3}^{*} \otimes\left(\frac{1}{2} e_{2}+a e_{3}\right) \\
& r_{30}=\left(a e_{1}+e_{2}+2 a e_{3}\right) \otimes e_{1}^{*}-\left(\frac{a}{2} e_{1}+\frac{1}{2} e_{2}+a e_{3}\right) \\
& \otimes e_{3}^{*}-e_{1}^{*} \otimes\left(a e_{1}+e_{2}+2 a e_{3}\right)+e_{3}^{*} \\
& \otimes\left(\frac{a}{2} e_{1}+\frac{1}{2} e_{2}+a e_{3}\right),
\end{aligned}
$$

$$
\begin{aligned}
r_{31} & =e_{2} \otimes e_{1}^{*}-\frac{1}{2} e_{2} \otimes e_{3}^{*}-e_{1}^{*} \otimes e_{2}+e_{3}^{*} \otimes \frac{1}{2} e_{2}, \\
r_{32} & =\left(a e_{1}+e_{2}+2(a+b) e_{3}\right) \otimes e_{1}^{*} \\
& +\left(a b e_{1}+b e_{2}-2\left(b^{2}+a b\right) e_{3}\right) \otimes e_{2}^{*} \\
& -\left(\frac{a}{2} e_{1}+\frac{1}{2} e_{2}+(a+b) e_{3}\right) \otimes e_{3}^{*}-e_{1}^{*} \\
& \otimes\left(a e_{1}+e_{2}+2(a+b) e_{3}\right)-e_{2}^{*} \\
& \otimes\left(a b e_{1}+b e_{2}-2\left(b^{2}+a b\right) e_{3}\right) \otimes e_{2}^{*}+e_{3}^{*} \\
& \otimes\left(\frac{a}{2} e_{1}+\frac{1}{2} e_{2}+(a+b) e_{3}\right) .
\end{aligned}
$$

One can check that all of the tensors above are solutions of the classical Yang-Baxter equation in $g \ltimes_{\mathrm{ad}^{*}} g^{*}$.

\section{Induced Left-Symmetric Algebras from Rota-Baxter Operators of Weight 0 on $g$}

A left-symmetric algebra structure on $g$ is a bilinear product $\cdot: g \otimes g \rightarrow g$ satisfying the condition

$$
x \cdot(y \cdot z)-(x \cdot y) \cdot z=y \cdot(x \cdot z)-(y \cdot x) \cdot z
$$

for all $x, y, z \in g$. There are many examples of Lie algebras which do not admit a left-symmetric product. For example, it is easy to see that there are no left-symmetric algebras with semisimple Lie algebra. Equation (31) implies that the commutators $[x, y]=x \cdot y-y \cdot x$ satisfy the Jacobi identity; that is to say each left-symmetric product has an associated commutation Lie algebra, which is called the subadjacent Lie algebra. If $R$ is a Rota-Baxter operator on a left-symmetric algebra, then $R$ is a solution of CYBE on its subadjacent Lie algebra [17]. Clearly, each associative algebra product is a leftsymmetric product. Given a Lie algebra $g$, it is a fundamental problem to decide whether $g$ admits a left-symmetric product and to give a classification of such products [18]. As an application of Yang-Baxter operators, we can use them to construct left-symmetric algebras with respect to $g_{6}$.

Lemma 4 (see [13]). Let $g$ be a Lie algebra; $P$ is called a solution of the classical Yang-Baxter equation. Define a new operation on $g$ by

$$
x * y=[P(x), y], \quad \forall x, y \in g .
$$

Then $(g, *)$ is a left-symmetric algebra.

According to Theorem 3 and Lemma 4, we can get some left-symmetric algebras of $g$.

Theorem 5. Some left-symmetric algebras of $g$ (of weight zero) are determined:

(1) $e_{3} * e_{1}=-e_{1}, e_{3} * e_{2}=a e_{1}, e_{3} * e_{3}=e_{1}+e_{3}$;

(2) $e_{2} * e_{1}=-a e_{1}, e_{2} * e_{2}=a^{2}\left(e_{1}+e_{3}\right), e_{2} * e_{3}=a\left(e_{1}+e_{3}\right)$, $e_{3} * e_{1}=-e_{1}, e_{3} * e_{2}=a\left(e_{1}+e_{3}\right), e_{3} * e_{3}=e_{1}+e_{3}$; 
(3) $e_{3} * e_{1}=-e_{1}, e_{3} * e_{3}=e_{1}+e_{3}$;

(4) $e_{2} * e_{1}=-a e_{1}, e_{2} * e_{2}=\left(a^{2}+a b\right) e_{1}+a^{2} e_{3}, e_{2} * e_{3}=$ $a\left(e_{1}+e_{3}\right), e_{3} * e_{1}=-e_{1}, e_{3} * e_{2}=(a+b) e_{1}+a e_{3}$, $e_{3} * e_{3}=e_{1}+e_{3}$

(5) $e_{2} * e_{2}=(a-1) e_{1}-e_{3}$;

(6) $e_{2} * e_{2}=e_{1}$;

(7) $e_{2} * e_{2}=-\left(e_{1}+e_{3}\right)$;

(8) $e_{2} * e_{2}=(a-b) e_{1}-b e_{3}, e_{3} * e_{2}=e_{1}$;

(9) $e_{2} * e_{2}=a e_{1}, e_{3} * e_{2}=e_{1}$;

(10) $e_{2} * e_{2}=-a\left(e_{1}+e_{3}\right), e_{3} * e_{2}=e_{1}$;

(11) $e_{3} * e_{2}=e_{1}$;

(12) $e_{2} * e_{1}=-e_{1}, e_{2} * e_{2}=(a-b) e_{1}-b e_{3}, e_{2} * e_{3}=e_{1}+e_{3}$;

(13) $e_{2} * e_{1}=-e_{1}, e_{2} * e_{2}=a e_{1}, e_{2} * e_{3}=e_{1}+e_{3}$;

(14) $e_{2} * e_{1}=-e_{1}, e_{2} * e_{2}=-a\left(e_{1}+e_{3}\right), e_{2} * e_{3}=e_{1}+e_{3}$;

(15) $e_{2} * e_{1}=-e_{1}, e_{2} * e_{3}=e_{1}+e_{3}$;

(16) $e_{1} * e_{2}=-\left(e_{1}+e_{3}\right), e_{2} * e_{2}=(a-b) e_{1}-b e_{3}, e_{3} * e_{2}=$ $e_{1}+e_{3}$

(17) $e_{1} * e_{2}=(a-1) e_{1}-e_{3}, e_{2} * e_{2}=-b\left(e_{1}+e_{3}\right), e_{3} * e_{2}=$ $(a-1)^{2} e_{1}-(a-1) e_{3}$;

(18) $e_{1} * e_{2}=(a-1) e_{1}-e_{3}, e_{2} * e_{2}=b e_{1}, e_{3} * e_{2}=$ $(a-1)^{2} e_{1}-(a-1) e_{3}$

(19) $e_{1} * e_{2}=-\left(e_{1}+e_{3}\right), e_{2} * e_{2}=-a\left(e_{1}+e_{3}\right), e_{3} * e_{2}=e_{1}+e_{3}$;

(20) $e_{1} * e_{2}=e_{1}+e_{3}, e_{2} * e_{2}=a e_{1}, e_{3} * e_{2}=e_{1}+e_{3}$;

(21) $e_{1} * e_{2}=(a-1) e_{1}-e_{3}, e_{3} * e_{2}=(a-1)^{2} e_{1}-(a-1) e_{3}$;

(22) $e_{1} * e_{2}=-\left(e_{1}+e_{3}\right), e_{3} * e_{2}=e_{1}+e_{3}$;

(23) $e_{1} * e_{2}=(a-1) e_{1}-e_{3}, e_{2} * e_{2}=(b-c) e_{1}-c e_{3}$, $e_{3} * e_{2}=(a-1)^{2} e_{1}-(a-1) e_{3} ;$

(24) $e_{1} * e_{2}=-(1 / 2) e_{1}-e_{3}, e_{2} * e_{2}=-a\left(e_{1}+e_{3}\right), e_{3} * e_{2}=$ $(1 / 4) e_{1}+(1 / 2) e_{3}$

(25) $e_{1} * e_{2}=-(1 / 2) e_{1}-e_{3}, e_{2} * e_{2}=a e_{1}, e_{3} * e_{2}=(1 / 4) e_{1}+$ $(1 / 2) e_{3}$

(26) $e_{1} * e_{2}=-(1 / 2) e_{1}-e_{3}, e_{3} * e_{2}=(1 / 4) e_{1}+(1 / 2) e_{3}$;

(27) $e_{1} * e_{2}=-(1 / 2) e_{1}-e_{3}, e_{2} * e_{2}=(a-b) e_{1}-b e_{3}$, $e_{3} * e_{2}=(1 / 4) e_{1}+(1 / 2) e_{3} ;$

(28) $e_{1} * e_{1}=-e_{1}, e_{1} * e_{2}=-2 a\left(e_{1}+e_{3}\right), e_{1} * e_{3}=e_{1}+e_{3}$, $e_{2} * e_{1}=-a e_{1}, e_{2} * e_{2}=-2 a^{2}\left(e_{1}+e_{3}\right), e_{2} * e_{3}=$ $a\left(e_{1}+e_{3}\right), e_{3} * e_{1}=(1 / 2) e_{1}, e_{3} * e_{2}=a\left(e_{1}+e_{3}\right)$, $e_{3} * e_{3}=-(1 / 2)\left(e_{1}+e_{3}\right)$;

(29) $e_{1} * e_{1}=-e_{1}, e_{1} * e_{2}=-a e_{1}-2 a e_{3}, e_{1} * e_{3}=e_{1}+e_{3}$, $e_{3} * e_{1}=(1 / 2) e_{1}, e_{3} * e_{2}=(a / 2) e_{1}+a e_{3}, e_{3} * e_{3}=$ $-(1 / 2)\left(e_{1}+e_{3}\right)$;

(30) $e_{1} * e_{3}=e_{1}+e_{3}, e_{3} * e_{3}=-(1 / 2)\left(e_{1}+e_{3}\right)$;

(31) $e_{1} * e_{1}=-e_{1}, e_{1} * e_{2}=-(a+2 b) e_{1}-2(a+b) e_{3}$, $e_{1} * e_{3}=e_{1}+e_{3}, e_{2} * e_{1}=-b e_{1}, e_{2} * e_{2}=\left(2 b^{2}+3 a b\right) e_{1}+$ $\left(2 b^{2}+2 a b\right) e_{3}, e_{2} * e_{3}=b\left(e_{1}+e_{3}\right), e_{3} * e_{1}=(1 / 2) e_{1}$, $e_{3} * e_{2}=(a / 2+b) e_{1}+(a+b) e_{3}, e_{3} * e_{3}=-(1 / 2)\left(e_{1}+e_{3}\right)$.

\section{Conflicts of Interest}

The authors declare no conflicts of interest.

\section{Acknowledgments}

This work is supported by the National Science Foundation of China (Grants nos. 11047030 and 11771122) and the Science and Technology Program of Henan Province (Grant no. 152300410061).

\section{References}

[1] G. Baxter, "An analytic problem whose solution follows from a simple algebraic identity," Pacific Journal of Mathematics, vol. 10, pp. 731-742, 1960.

[2] K. Ebrahimi-Fard, "Loday-type algebras and the Rota-Baxter relation," Letters in Mathematical Physics, vol. 61, no. 2, pp. 139$147,2002$.

[3] L. Guo, An Introdction to Roto-Baxter Algebra, International Press, Beijing, China, 2012.

[4] L. Guo and W. Keigher, "Baxter algebras and shuffle products," Advances in Mathematics, vol. 150, no. 1, pp. 117-149, 2000.

[5] R. Bai, L. Guo, J. Li, and Y. Wu, "Rota-Baxter 3-Lie algebras," Journal of Mathematical Physics, vol. 54, no. 6, Article ID 063504, 2013.

[6] A. A. Belavin and V. G. Drinfel'd, "Solutions of the classical Yang - Baxter equation for simple Lie algebras," Functional Analysis and its Applications, vol. 16, no. 3, pp. 159-180, 1982.

[7] Y. Cheng and Y. Su, "Quantum deformations of the HeisenbergVirasoro algebra," Algebra Colloquium, vol. 20, no. 2, pp. 299308, 2013.

[8] A. Makhlouf and D. Yau, "Rota-Baxter Hom-Lie-admissible algebras," Communications in Algebra, vol. 42, no. 3, pp. 12311257, 2014.

[9] X. Tang, Y. Zhang, and Q. Sun, "Rota-Baxter operators on 4-dimensional complex simple associative algebras," Applied Mathematics and Computation, vol. 229, pp. 173-186, 2014.

[10] Y. Cheng and Y. Shi, "Lie bialgebra structures on the $q$-analog Virasoro-like algebras," Communications in Algebra, vol. 37, no. 4, pp. 1264-1274, 2009.

[11] P. Etingof, T. Schedler, and A. Soloviev, "Set-theoretical solutions to the quantum Yang-Baxter equation," Duke Mathematical Journal, vol. 100, no. 2, pp. 169-209, 1999.

[12] J. Pei, C. Bai, and L. Guo, "Rota-Baxter operators on $s l(2, \mathbb{C})$ and solutions of the classical Yang-Baxter equation," Journal of Mathematical Physics, vol. 55, no. 2, Article ID 021701, 2014.

[13] X. Li, D. Hou, and C. Bai, "Rota-Baxter operators on pre-Lie algebras," Journal of Nonlinear Mathematical Physics, vol. 14, no. 2, pp. 269-289, 2007.

[14] M. A. Semenov-Tyan-Shanskii, "What is a classical r-matrix?" Functional Analysis and its Applications, vol. 17, no. 4, pp. 259272, 1983.

[15] C. Bai, "A unified algebraic approach to the classical YangBaxter equation," Journal of Physics A: Mathematical and General, vol. 40, no. 36, pp. 11073-11082, 2007.

[16] C. Bai, L. Guo, and X. Ni, "Generalizations of the classical YangBaxter equation and 0 -operators," Journal of Mathematical Physics, vol. 52, no. 6, Article ID 063515, 2011.

[17] H. An and C. Bai, "From Rota-Baxter algebras to pre-Lie algebras," Journal of Physics A: Mathematical and Theoretical, vol. 41, no. 1, Article ID 015201, 2008.

[18] O. Baues, "Left-symmetric algebras for $g l(n), "$ Transactions of the American Mathematical Society, vol. 351, no. 7, pp. 29792996, 1999. 


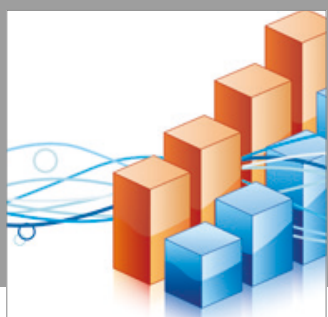

Advances in

Operations Research

vatersals

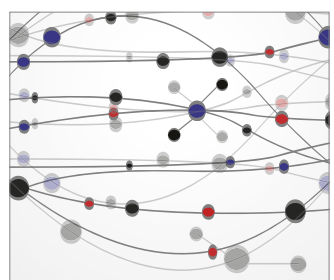

\section{The Scientific} World Journal
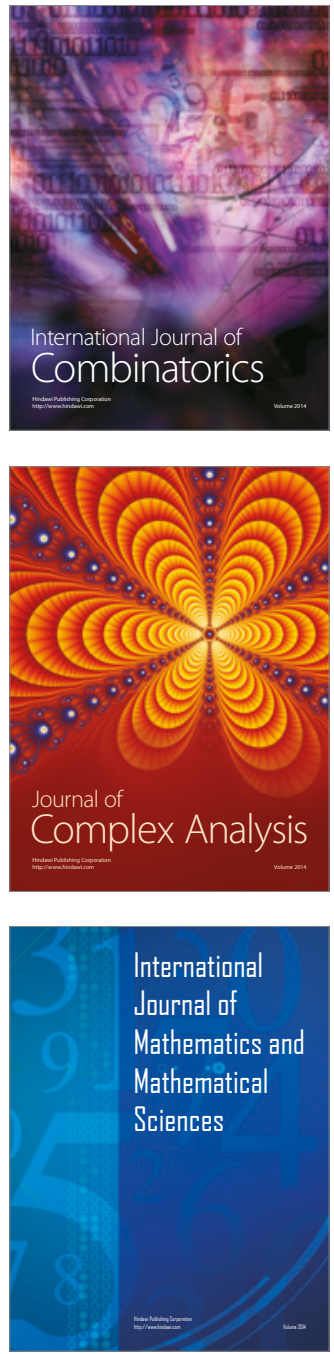
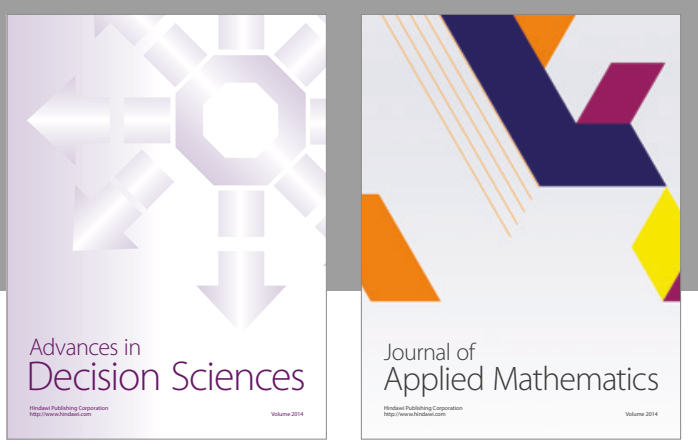

Algebra

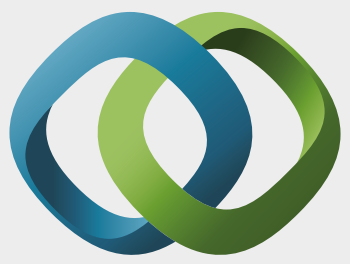

\section{Hindawi}

Submit your manuscripts at

https://www.hindawi.com
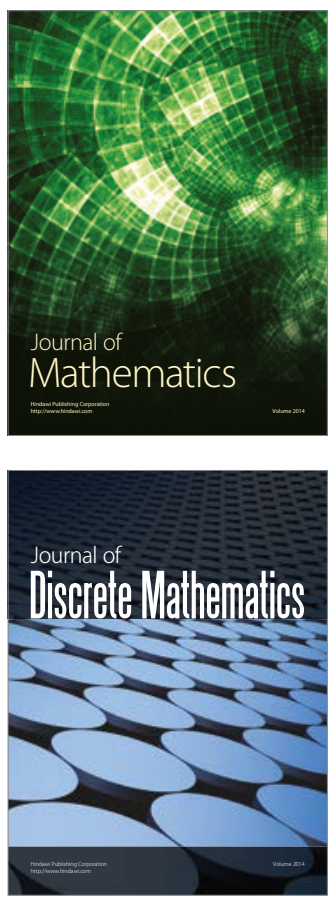

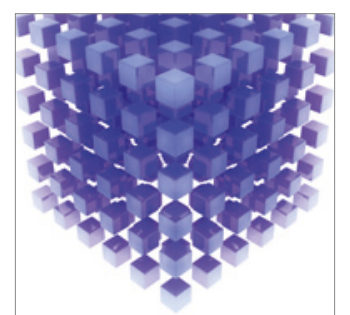

Mathematical Problems in Engineering
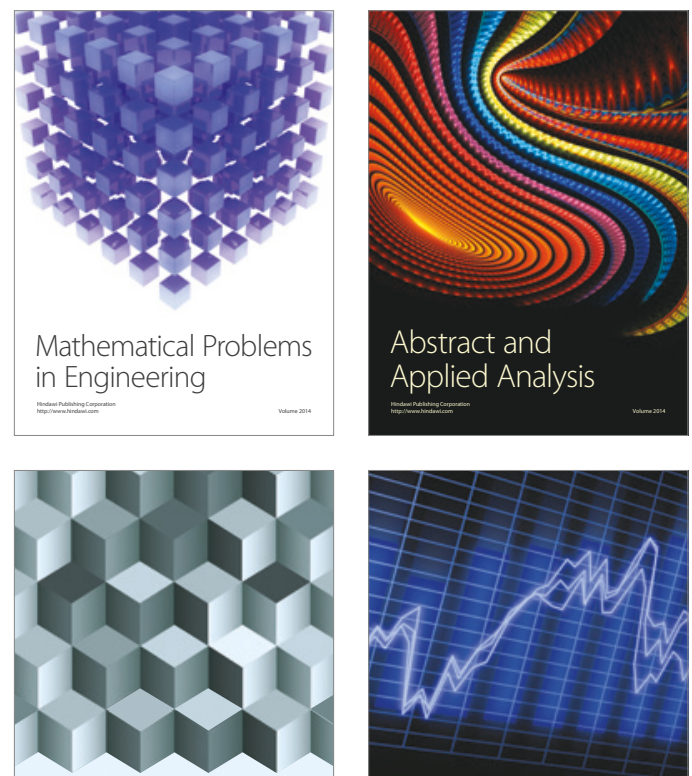

Journal of

Function Spaces

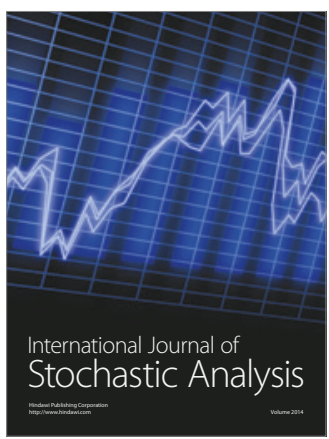

Probability and Statistics
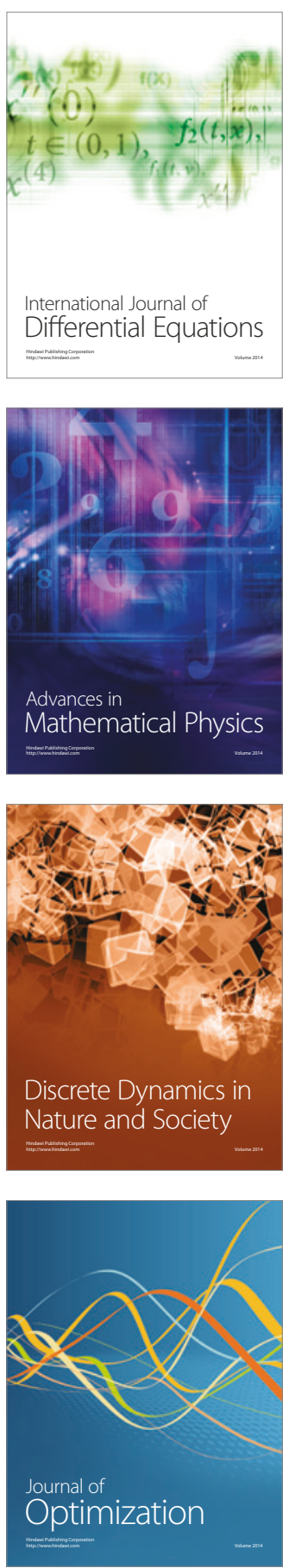